\title{
The Impact of Fading on the Outage Probability in Cognitive Radio Networks
}

\author{
Yaobin $\mathrm{Wen}^{\dagger}$, Sergey Loyka ${ }^{\dagger}$ and Abbas Yongacoglu ${ }^{\dagger}$
}

\begin{abstract}
This paper analyzes the outage probability in cognitive radio networks, based on the Poisson point process model of node spatial distribution and the standard propagation path loss model, including Rayleigh and log-normal fading. To make the analysis tractable, all possible scenarios are classified into three cases based on typical outage events. When the average number of nodes in the forbidden region is much smaller than unity, the aggregate interference can be well approximated by the nearest node for both non-fading and fading scenarios (the nearest node dominates the outage performance). When the average number of nodes in the forbidden region is greater than unity, the aggregate interference can be well approximated by a Gaussian random variable for non-fading scenario (many nodes contribute to outage events, rather than a single dominant one). This approximation also applies to the fading scenario, but its accuracy is a bit worse at the transition region. An alternative approximation is proposed, which is accurate for any outage probability. When the average number of nodes in the forbidden region is slightly smaller than unity, neither the nearest node approximation nor the Gaussian one is accurate for the nonfading scenario (since only a few near-by nodes are dominant), and finding an accurate approximation for the outage probability in this case is an open problem. The alternative approximation above is accurate for the fading scenario. All approximations are validated via Monte-Carlo simulations.
\end{abstract}

\section{INTRODUCTION}

As higher data rate services are required in wireless communications over a limited spectrum available, there is a need for more spectrum efficiency. To overcome the overcrowded spectrum problem and use spectrum more efficiently, Cognitive Radio (CR) suggests allowing secondary users (SU) to share the spectrum which is not currently used by the primary user (PU)[1]. Due to the uncertainty of SU number and locations, the PU performance may be seriously affected by the aggregate interference generated by SUs, so its accurate modeling is important to design cognitive radio networks and also to estimate potential benefits.

To model the aggregate interference in a wireless network, we have to properly choose node spatial distribution and propagation path loss models. The most popular spatial distribution model is a Poisson point process on a plane. Based on this model and average propagation path loss model, Sousa and Silvester [2] studied the aggregate interference power. They obtained its characteristic function $(\mathrm{CF})$ and concluded that the aggregate interference power is an $\alpha$-stable random variable. Sousa [3] extended that model and studied the aggregate

$\dagger$ Y. Wen, S. Loyka and A. Yongacoglu are with the School of Information Technology and Engineering, University of Ottawa, Ontario, Canada, K1N 6N5, e-mails: ywen027@uottawa.ca, sergey.loyka@ieee.org, yongacog@site.uottawa.ca. interference as a random vector at the output of receiver correlators, and concluding that the aggregate interference is a symmetric $\alpha$-stable random vector. Using the multivariate Lepage series representation, Ilow and Hatzinakos [4] obtained the $\mathrm{CF}$ of the aggregate interference according to a Poisson point process of node locations in the plane/volume, including the log-normal and Rayleigh fading effects and concluding that the aggregate interference is a spherically symmetric $\alpha$-stable random vector. Mordachev and Loyka [5] studied the tradeoff of the outage probability and the node density in wireless networks also based on the Poisson point process and the average path loss, but including different fading models and interference cancellation mechanisms as well. By studying the tail of the aggregate interference distribution, they found that, at the low outage region, the aggregate interference is dominated by the nearest one. Based on this, compact, closedform expressions for outage probability were obtained and a number of insights were pointed out. Ghasemi and Sousa [6] studied the aggregate interference in cognitive radio networks based on the Poisson point process, the average path loss and different fading models. Using Campell's theorem, the CF and cumulants of the aggregate interference power was obtained, and the an approximation of the outage probability is derived based on the cumulants. The effect of cooperative sensing on the distribution of the aggregate interference power for i.i.d. fading channels has also been studied.

In a typical cognitive radio network, SUs inside of a forbidden region around the PU are not allowed to transmit (while details of a typical CR protocol may vary, a forbidden region is always present), so that the distribution of the aggregate interference is not $\alpha$ - stable any more and the models of [2]-[5] cannot be applied directly. On the other hand, the approximation in [6] uses only first three cumulants so that its accuracy is not high when the forbidden region is small and the SU node density is low.

To overcome these limitations, we develop a new method to study the distribution of aggregate interference and the outage probability in cognitive radio networks. To make the analysis tractable, all possible scenarios are classified into three cases, based on typical (dominant) outage events (i.e. when the aggregate interference at the PU receiver exceeds a threshold):

- Case 1: when the average number of SU nodes in the forbidden region is much smaller than unity, a dominant outage event is when the nearest node interference exceeds the threshold, and the aggregate interference can be well approximated by the interference of the nearest node. We derive a closed-form outage probability expression in terms 
of the threshold interference to noise ratio (INR).

- Case 2: when the average number of nodes in the forbidden region is greater than unity, the combined interference from many nodes exceeding the threshold is a typical outage event. In this case, the aggregate interference can be approximated by a Gaussian random variable, for which we present a simple way to find its cumulants.

- Case 3: when the average number of nodes in the forbidden region is only slightly smaller than unity, a typical outage event is when the combination of a few nearest node interference exceeds the threshold. Neither the nearest node approximation nor Gaussian one is accurate in this case. Finding an accurate approximation in this case is an open problem.

The three cases above also apply to the Rayleigh and lognormal fading scenarios with some minor modifications (some approximations and cumulants are different - see Section IV and $\mathrm{V}$ ), so that this typical outage event classification is robust to the model details and also suggests a way to reduce the outage probability significantly.

The paper is organized as follows. In Section II, the node spatial distribution model, the CR protocol and propagation path loss model are introduced. Section III, IV and V analyze the distribution of the aggregate interference and outage probability in terms of the INR in non-fading, Rayleigh fading and log-normal fading scenarios respectively. Monte-Carlo simulation results validate the analysis and approximations.

\section{SYSTEM MODEL}

We consider a cognitive radio network which contains a primary user (PU) receiver and many secondary users' (SU) transmitters (nodes) on a plane. The PU is located at the origin. The SUs are randomly located according to a Poisson point process. The density of SUs is $\lambda\left[\right.$ nodes $\left./ \mathrm{m}^{2}\right]$. Interference from the $\mathrm{SU}$ nodes outside the circle of a certain radius $R_{\max }$ is assumed to be negligible (alternatively, no SUs are located outside of this circle). The CR protocol is that all SUs which are inside of a forbidden region, i.e. the circle of the radius $R_{s}$ centered on the PU, cease their transmissions so that some protection to the PU is provided.

We assume the desired signal, interferences and noise are independent of each other. The received power of the PU can be expressed as:

$$
P_{P U}=P_{d}+\sum_{i=1}^{N} I_{i}+P_{0}
$$

where $P_{d}$ is the desired signal power; $I_{i}$ is the interference signal power coming from the $i^{\text {th }}$ node; $P_{0}$ is the noise power; $N$ is a Poisson random variable which denotes the number of nodes in the ring between circles of the radii $R_{s}$ and $R_{\max }$, i.e. the potential interference zone. We follow the standard propagation model, which was used in [5]. The power at the receiver antenna output coming from a transmitter of power $P_{t}$ is $P_{r}=P_{t} G_{t} G_{r} g$, where $G_{t}$ and $G_{r}$ are the transmitter and receiver antenna gains; $g$ is the propagation path loss, $g=g_{a} g_{l} g_{s}$, where $g_{a}$ is the average path loss, $g_{l}$ is the largescale fading, and $g_{s}$ is the small-scale fading; $g_{a}=a_{\nu} r^{-\nu}$, where $\nu$ is the path loss exponent, $r$ is the distance between the transmitter and receiver, and $a_{\nu}$ is a constant independent of $r$. For simplicity, we assume the transmitter and receiver antennas are isotropic with unity gain, so that $P_{r}=P_{t} g$, and that all SUs transmit at the same constant power level $P_{s}$. In the nonfading scenario, the $i^{t h} \mathrm{SU}$ generates the interference power $I_{a i}=P_{s} a_{\nu} r_{i}^{-\nu}$ at the PU receiver, where $r_{i}$ is the distance between the $i^{\text {th }} \mathrm{SU}$ and the PU. Without loss of generality, we normalize $P_{s} a_{\nu}=1$, so $I_{a i}=r_{i}^{-\nu}$.

The non-fading scenario is considered in Section III, and the effect of fading is included in Section IV and V.

\section{OUTAGE PROBABILITY: THE NON-FADING SCENARIO}

When signal to interference plus noise ratio (SINR) is less than a certain threshold $\eta$, there is significant performance degradation of a wireless link and it is considered to be in outage. The probability of SINR being less than $\eta$ is an outage probability. When the signal and noise powers are fixed, the outage probability is the probability of aggregate interference $I_{a g}$ exceeding the threshold $I_{t h}=P_{d} / \eta-P_{0}$,

$$
P_{\text {out }}=\operatorname{Pr}\{\operatorname{SINR}<\eta\}=\operatorname{Pr}\left\{I_{a g}>I_{\text {th }}\right\}
$$

Defining the interference to noise ratio (INR) as

$$
\gamma=\frac{\sum_{i=1}^{N} I_{a i}}{P_{0}}
$$

its threshold value is $D=I_{t h} / P_{0}$, so that the outage probability is:

$$
P_{\text {out }}=\operatorname{Pr}\{\gamma>D\}=1-F(D)
$$

where $F(D)$ is the CDF of the INR. The interference from a single $\mathrm{SU}$ in the disk of the radius $R(D)=\left(D P_{0}\right)^{-1 / \nu}$ results in the INR greater than $D$, so that $P_{\text {out }}$ is equivalently a probability of having at least one SU in this disk.

When all SUs are allowed to transmit, $R_{s}=0$, the scenario is the same as in [5], so that from [[5] Theorem 1],

$$
\lim _{x \rightarrow \infty} \frac{\operatorname{Pr}\left\{\sum_{i=1}^{N} I_{a i}>x\right\}}{\operatorname{Pr}\left\{I_{a 1}>x\right\}}=1, I_{a 1} \geq \cdots \geq I_{a N},
$$

where $I_{a 1}$ is the strongest (nearest node) interference, and, at the low outage region (large $x$ ), the aggregate interference is dominated by the nearest one, $\operatorname{Pr}\left\{\sum_{i=1}^{N} I_{a i}>x\right\} \approx$ $\operatorname{Pr}\left\{I_{a 1}>x\right\}$, so that the outage probability can be approximated as in [5],

$$
P_{\text {out }} \approx \begin{cases}1, & D \leq D_{0} \\ \bar{N}_{0} D^{-2 / \nu}, & D>D_{0}\end{cases}
$$

where $\bar{N}_{0}=\pi \lambda R_{0}^{2}$ is the average number of nodes in the disk of radius $R_{0}=P_{0}^{-1 / \nu}$ (the interference level is below the noise level outside the circle of the radius $R_{0}$; this disk was termed "potential interference zone" in [5]), $D_{0}=\bar{N}_{0}^{\nu / 2}$ is a critical value which separates the high and low outage probability regions. It corresponds to on average one SU being in the disk of the radius $R\left(D_{0}\right)$, so that the outage probability is high if $D \leq D_{0}$, since $R(D) \geq R\left(D_{0}\right)$ and there is a high probability of having at least one $\mathrm{SU}$ in the disk of radius $R(D)$. 
When the CR protocol is implemented, all SU nodes inside the forbidden region $R_{s}$ cease their transmissions. The interference generated by a single node can not exceed $I_{\max }=R_{s}^{-\nu}$, i.e. the value coming from an active node at the closest possible distance, so that the maximum INR from a single node is $D_{\max }=I_{\max } / P_{0}$. Thus, the results in (5) and (6) do not apply anymore.

To obtain similar approximations for the CR network, we classify all possible scenarios into three different cases based on typical outage events, which are further linked to $R\left(D_{0}\right)$ and $R_{s}$.

Case 1: When $R_{s} \ll R\left(D_{0}\right)$, the aggregate interference is dominated by the nearest node one for $D<D_{\max }$ : $\operatorname{Pr}\left\{\sum_{i=1}^{N} I_{a i}>D P_{0}\right\} \approx \operatorname{Pr}\left\{I_{a 1}>D P_{0}\right\}$.

To demonstrate that the nearest interference is indeed dominant in this case, we consider two subcases:

- Case 1.A: $R_{s} \ll R(D)$.

- Case 1.B: $R_{s}<R(D)$ but not $R_{s} \ll R(D)$.

Let $\operatorname{Ring}\left(r_{1}, r_{2}\right)$ be a ring between the circles of the radii $r_{2} \geq r_{1}$, and $\operatorname{Ring}\left(R_{s}, R(D)\right)$ be a first ring, and $\operatorname{Ring}(R(D /(k-1)), R(D / k)), k=2,3, \ldots$ be the $k^{t h}$ ring, so that the combined interference to noise ratio from $k$ nodes in this ring exceeds $D$, i.e. causes an outage event.

For Case 1.A, $\pi R^{2}(D)>>\pi R_{s}^{2}$, so that $\pi\left(R^{2}(D)-R_{s}^{2}\right) \approx$ $\pi R^{2}(D)$, and hence the probability to have at least one node in $\operatorname{Ring}\left(R_{s}, R(D)\right)$, which is the outage probability, is roughly the same as that for $\operatorname{Ring}(0, R(D))$, so that $\operatorname{Pr}\left\{\sum_{i=1}^{N} I_{a i}>D P_{0}\right\} \approx \operatorname{Pr}\left\{I_{a 1}>D P_{0}\right\}$ and the corresponding results in [5] can be used as long as $D<D_{\max }$.

For Case 1.B, it can be shown that the nearest interference is still dominant, so that $P_{\text {out }} \approx 1-\exp \left(\bar{N}_{0}\left(D_{\max }^{-2 / \nu}-D^{-2 / \nu}\right)\right)$ when $D<D_{\max }$.

When $D>D_{\max }$, i.e. $R_{s}>R(D)$, the typical outage event is $n+2$ or more nodes being in $\operatorname{Ring}\left(R_{s}, R(D /(n+2))\right.$, where $n=f l o o r\left(D / D_{\max }\right)$, and the aggregate interference is dominated by a few nearest nodes. However, since the outage probability is very small and drops very fast in this region, $P_{\text {out }} \approx 0$ is a reasonable approximation (see Fig. 1).

Finally, the outage probability in Case 1 can be approximated as

$$
P_{\text {out }} \approx \begin{cases}1-\exp \left[\bar{N}_{0}\left(D_{\max }^{\frac{-2}{\nu}}-D^{\frac{-2}{\nu}}\right)\right] & D<D_{\max } \\ 0 & D \geq D_{\max }\end{cases}
$$

Note that it is determined by only three critical parameters: $\nu$, $D_{\max }$ and $\bar{N}_{0}$. When $D \ll D_{\max }$, the approximation in (6) applies, and $P_{\text {out }} \approx 1$ when $D \leq D_{0}$.

Fig. 1 validates the approximation above. Clearly, there are 3 different regions: (i) when $D<D_{0}, P_{\text {out }}$ is high; (ii) when $D_{0}<D<D_{\max }$, the aggregate interference is dominated by the nearest node one; (iii) when $D>D_{\max }, P_{\text {out }}$ is very small and drops very fast. On the other hand, Gaussian approximation, which was used in [6], is not accurate in this case.

Case 2: The aggregate interference is closely approximated by a Gaussian random variable, $\sum_{i=1}^{N} I_{a i} \sim \mathcal{N}$, when $R_{s}>$ $R\left(D_{0}\right)$.

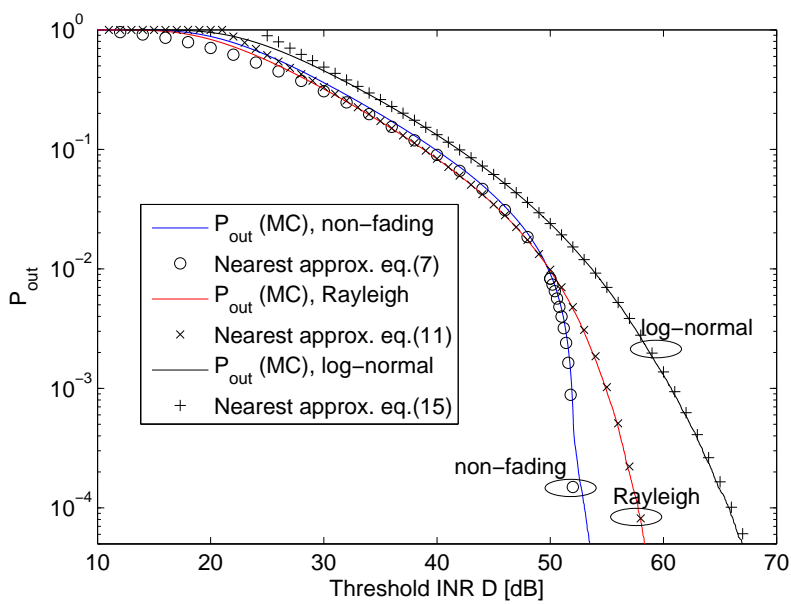

Fig. 1. Outage probability for Case 1. $\nu=4, R_{s}=10 \mathrm{~m}, R\left(D_{0}\right)=$ $56.4 \mathrm{~m}, R_{0}=200 \mathrm{~m}, R_{\max }=10^{3} \mathrm{~m}, \lambda=10^{-4}\left[\right.$ nodes $\left./ \mathrm{m}^{2}\right], D_{0}=$ $22 \mathrm{~dB}, D_{\max }=52 \mathrm{~dB}, \sigma=1.38$. MC denotes Monte-carlo simulations. Note that the nearest node approximation works well in the whole INR range for all fading and non-fading scenarios. While fading has in general negative effect on $P_{\text {out }}$, the impact of log-normal shadowing is more pronounced, especially at $D>D_{\max }$.

Since $R_{s}>R\left(D_{0}\right)$, the average number of nodes in the disk of radius $R_{s}$ is larger than one. When $R(D) \geq R_{s}$, the average number of nodes in first few rings is not small, so a typical outage event is when aggregate interference from many nodes in these rings exceeds the threshold $I_{t h}$. When $R(D)<R_{s}$, many nodes in a few nearest rings are required to produce an outage event since the single-node INR can not exceed $D_{\max }$. Thus, the aggregate interference in Case 2 can be well approximated by a Gaussian random variable based on the central limit theorem.

From the system model, random variable $I_{i}$ represents the interference coming from $i$-th node without ordering. Poisson point distribution has a property that points in any nonoverlapping regions of space are statistically independent, so that, different $I_{i}$ are independent of each other. The aggregate interference $I_{a g}=\sum_{i=1}^{N} I_{i}$ so that its cumulants can be found using the distribution of $I_{i}$. For sufficiently large $R_{\max }$, these cumulants are:

$$
\kappa_{n}=\frac{2 \pi \lambda R_{s}^{2-n \nu}}{n \nu-2}, \nu>2
$$

Using the first two cumulants, the outage probability can be approximated via the Gaussian distribution,

$$
P_{\text {out }}=\operatorname{Pr}\{\gamma>D\} \approx Q\left(\frac{D P_{0}-\kappa_{1}}{\sqrt{\kappa_{2}}}\right)
$$

where $Q(x)=1 / \sqrt{2 \pi} \int_{x}^{\infty} \exp \left(-u^{2} / 2\right) d u$ is the $\mathrm{Q}$ function. When higher order cumulants of $I_{a g}$ are used, more accurate approximations, e.g. an Edgeworth expansion, may be derived [6].

Monte-Carlo simulations show that the approximation in (9) is sufficiently accurate in this case.

Case 3: $R_{s}<R\left(D_{0}\right)$ but not $R_{s} \ll R\left(D_{0}\right)$; neither the nearest node approximation nor Gaussian one is accurate.

In this case, a typical outage event is when the combination of interference from a few nearest nodes exceeds the threshold. 
The nearest node approximation is not accurate (since several nodes are involved), and gives us a lower bound for the outage probability. On the other hand, the number of the nodes involved in a typical outage is not large enough to apply the central limit theorem, so that Gaussian approximation is not accurate too. Obtaining an accurate approximation in this case is an open problem.

\section{IMPACT OF RAYLEIGH FADING}

In this section, we study the impact of Rayleigh fading on the aggregate interference distribution. Let us consider the ordered average interference power $I_{a 1} \geq I_{a 2} \geq \cdots \geq I_{a N}$ which are further subjected to Rayleigh fading so that the fading received powers are $I_{s i}=g_{s i} I_{a i}$, where $g_{s i}$ are the Rayleigh fading factors, assumed to be i.i.d, with the standard pdf $f_{g s}(x)=e^{-x}$. Here, we also consider three typical cases.

Case 1: $R_{s} \ll R\left(D_{0}\right)$; the aggregate interference is dominated by the nearest node: $\operatorname{Pr}\left\{\sum_{i=1}^{N} I_{s i}>D P_{0}\right\} \approx$ $\operatorname{Pr}\left\{I_{s 1}>D P_{0}\right\}$.

When $R_{s} \ll R(D)$, this case reduces to the corresponding no-CR scenario in [5], so that the nearest node is dominant, $\operatorname{Pr}\left\{\sum_{i=1}^{N} I_{s i}>D P_{0}\right\} \approx \operatorname{Pr}\left\{I_{s 1}>D P_{0}\right\}$. When $R_{s}$ is not much smaller than $R(D)$, on the other hand, numerical experimentation indicates that the nearest node is still dominant and the distribution tail follows the fading distribution, see Fig. 1 (this is also consistent with the large deviation theory). Therefore, we proceed to find the CCDF of the nearest node interference, which will serve as an approximation to the outage probability.

The nearest INR is $d_{s}=I_{s 1} / P_{0}=g_{s 1} I_{a 1} / P_{0}$, and its CCDF is

$$
\begin{aligned}
\operatorname{Pr}\left\{d_{s}>D\right\} & \approx\left[1-\exp \left(\frac{\bar{N}_{0}}{D_{\max }^{2 / \nu}}\right)\right] \exp \left(-\frac{D}{D_{\max }}\right) \\
& +\exp \left(\frac{\bar{N}_{0}}{D_{\max }^{2 / \nu}}\right) \frac{\bar{N}_{0}}{D^{2 / \nu}} \Gamma\left(\frac{2}{\nu}+1, \frac{D}{D_{\max }}\right)
\end{aligned}
$$

where $\Gamma(a, x)=\int_{x}^{\infty} t^{a-1} e^{-t} d t$ is incomplete Gamma function. The approximation in (10) holds at the low outage region (see Appendix for the proof).

The outage probability can now be approximated as:

$$
P_{\text {out }} \approx\left\{\begin{array}{lc}
1, & D<D_{0} \\
\text { eq. }(10), & D \geq D_{0}
\end{array}\right.
$$

When $D_{0} \leq D \ll D_{\max }$, (10) can be approximated by $\Gamma(2 / \nu+1) \bar{N}_{0} D^{-2 / \nu}$, and it is same as (33) in [5]. When $D \gg D_{\max }$, (10) can be approximated by

$$
\left[1-\left(1-\bar{N}_{0} / D_{\max }^{2 / \nu}\right) \exp \left(\bar{N}_{0} / D_{\max }^{2 / \nu}\right)\right] \exp \left(-D / D_{\max }\right),
$$

and the outage probability is dominated by Rayleigh fading and decreases as $\exp \left(-D / D_{\max }\right)$ in very low outage region.

Fig. 1 validates the approximation in (11). There are four different regions: (i) when $D \leq D_{0}, P_{\text {out }}$ is high; (ii) when $D_{0}<D \ll D_{\max }$, the effect of Rayleigh fading is the multiplicative shift by a constant factor $\Gamma(2 / \nu+1)$ of the non-fading case; (iii) when $D \gg D_{\max }$, the outage probability is dominated by the nearest node in a positive fading state $\left(g_{s}>1\right)$, and decreases as $\exp \left(-D / D_{\max }\right)$; (iv) the region between $D \ll D_{\max }$ and $D \gg D_{\max }$ is the transitional region. Clearly, the outage probability is well approximated by the distribution in (10) when $D>D_{0}$.

Case 2: When $R_{s}>R\left(D_{0}\right)$, the aggregate interference is approximated by a Gaussian random variable, $\sum_{i=1}^{N} I_{s i} \sim \mathcal{N}$, unless $D \gg D_{\max }$.

When $R_{s}>R\left(D_{0}\right)$, a typical outage event in the Rayleigh fading scenario is when the aggregate interference from many nodes exceeds the threshold $I_{t h}$, so that it can still be approximated by a Gaussian random variable. The rationale for this follows that of the non-fading Case 2. However, while the Gaussian approximation is accurate when $D$ is less or slightly higher than $D_{\max }$ and predicts well the sharp threshold behavior of $P_{\text {out }}$ (see Fig. 2), it is less accurate when $D \gg D_{\max }$.

To evaluate the cumulants of the aggregate interference needed for the Gaussian approximation, we consider unordered fading interference $I_{s}=I \cdot g_{s}$, where $I$ is the average interference coming from a randomly-selected node. Since the PDF of $g_{s}$ is $f_{g s}(x)=e^{-x}$, and since $I$ and $g_{s}$ are independent of each other, the $n^{\text {th }}$ moment of $I_{f}$ is $\mu_{n}=E\left[I_{f}^{n}\right]=E\left[I^{n}\right] E\left[g_{s}^{n}\right]$. Since $E\left[g_{s}^{n}\right]=n$ ! and $\kappa_{n}=\mu_{n}-\sum_{k=1}^{n-1}\left(\begin{array}{c}n-1 \\ k-1\end{array}\right) \kappa_{k} \mu_{n-k}$ $\left(\kappa_{1}=\mu_{1}\right)$, the cumulants of $I_{a g}$ for large $R_{\max }$ can be shown to be:

$$
\kappa_{n}=n ! \frac{2 \pi \lambda R_{s}^{2-n \nu}}{n \nu-2}, \nu>2
$$

and, using these cumulants, the outage probability can be approximated as in (9).

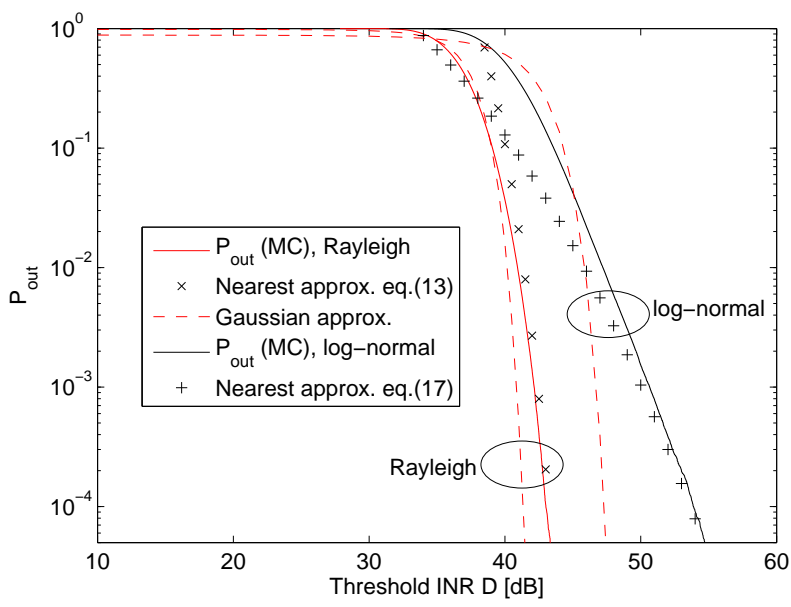

Fig. 2. Outage probability for Case $2 ; \nu=4, R_{s}=32 m, R\left(D_{0}\right)=18 m$, $R_{0}=200 \mathrm{~m}, R_{\max }=10^{3} \mathrm{~m}, \lambda=10^{-3}\left[\right.$ nodes $\left./ \mathrm{m}^{2}\right], D_{\max }=32 \mathrm{~dB}$, $\sigma=1.38$. MC denotes Monte-Carlo simulations; the Gaussian approximation is as in (9). While Rayleigh fading has a minor effect on $P_{\text {out }}$ (the nonfading curve is very close to the Rayleigh one and is not shown), log-normal shadowing has a significant impact on $P_{\text {out }}$.

Fig. 2 shows $P_{\text {out }}$ for Case 2. Clearly, the aggregate interference can be approximated by Gaussian approximation, but it is not very accurate when $D$ is close or exceeds $D_{\max }$ because of the effect of Rayleigh fading (single node can still cause an outage when it is in a positive fading state) and it decreases as $\exp \left(-D / D_{\max }\right)$ in this region (the positive tail of Rayleigh fading) according to (10). In fact, the nearest node 
approximation in (10) combined with the fact that $P_{\text {out }} \leq 1$ provides an approximation which is surprisingly accurate over the whole INR range,

$$
P_{\text {out }} \approx \min \{1 \text {, eq. }(10)\}
$$

We attribute this to the fact that, while many nodes contribute to the typical outage event before the steep transition region (so that Gaussian approximation is appropriate), it is a few nearest nodes plus positive fading that is dominant in the steep transition region.

Case 3: $R_{s}<R\left(D_{0}\right)$ but not $R_{s} \ll R\left(D_{0}\right)$ The nearest node approximation in (13) works well in this case as well, and the reason is same as in the previous case.

\section{IMPACT OF LOG-NORMAL FADING}

The ordered average interference powers $I_{a i}$ are further subjected to log-normal fading so that the received powers are $I_{l i}=g_{l i} I_{a i}$, where $g_{l i}$ are the log-normal fading factors, assumed to be i.i.d, with the pdf

$$
f_{g l}(x)=\frac{1}{\sqrt{2 \pi} x \sigma} \exp \left[\frac{-\ln ^{2} x}{2 \sigma^{2}}\right],
$$

where $\sigma$ is the standard deviation of $\ln x$ in natural units. Here, we also consider three typical cases based on typical outage events.

Case 1: $R_{s} \ll R\left(D_{0}\right)$; the aggregate interference is dominated by the nearest node: $\operatorname{Pr}\left\{\sum_{i=1}^{N} I_{l i}>D P_{0}\right\} \approx$ $\operatorname{Pr}\left\{I_{l 1}>D P_{0}\right\}$.

Similar as for the Rayleigh fading scenario, the nearest node is still dominant, so that the outage probability can be approximated by the CCDF of its received power,

$$
\begin{aligned}
\operatorname{Pr}\left\{d_{s}>D\right\} & \approx \frac{\bar{N}_{0}}{D^{2 / \nu}} \exp \left(\frac{2 \sigma^{2}}{\nu^{2}}\right) Q\left(\frac{\ln \left(D / D_{\max }\right)}{\sigma}-\frac{2 \sigma}{\nu}\right) \\
& -\frac{\bar{N}_{0}}{D_{\max }^{2 / \nu}} Q\left(\frac{\ln \left(D / D_{\max }\right)}{\sigma}\right)
\end{aligned}
$$

The approximation in (14) holds at the low outage region (see Appendix for the proof). The outage probability can now be approximated as:

$$
P_{\text {out }} \approx\left\{\begin{array}{lc}
1, & D<D_{0} \\
\text { eq.(14), } & D \geq D_{0}
\end{array}\right.
$$

Fig. 1 validates the approximation in (15).

Case 2: When $R_{s}>R\left(D_{0}\right)$, the aggregate interference is approximated by a Gaussian random variable, $\sum_{i=1}^{N} I_{l i} \sim \mathcal{N}$, unless $D \gg D_{\max }$.

When $R_{s}>R\left(D_{0}\right)$, similar as for the Rayleigh fading scenario, the aggregate interference is dominated by many nodes, not just the nearest one, so that Gaussian approximation is appropriate. The cumulants of $I_{a g}$ for large $R_{\max }$ can be shown to be:

$$
\kappa_{n}=\exp \left(\frac{n^{2} \sigma^{2}}{2}\right) \frac{2 \pi \lambda R_{s}^{2-n \nu}}{n \nu-2}, \nu>2
$$

and, using these cumulants, the outage probability can be approximated as in (9).
Fig. 2 shows $P_{\text {out }}$ for Case 2. Clearly, Gaussian approximation is accurate unless $D \gg D_{\max }$. In fact, the nearest node approximation in (14) combined with the fact that $P_{\text {out }} \leq 1$ is reasonably accurate over the whole INR range in this case,

$$
P_{\text {out }} \approx \min \{1 \text {, eq. }(14)\}
$$

Since the tail of the log-normal distribution is much heavier than the Rayleigh one, there are a few dominant nodes at the distribution tail in the former case unless $R_{s} \gg R\left(D_{0}\right)$, so that the Gaussian approximation is not accurate there, as Fig. 2 demonstrates. On the other hand, when $R_{s} \gg R\left(D_{0}\right)$, the Gaussian approximation becomes accurate because the probability of a few nodes causing an outage is very small, and the typical outage event is due to the combination of many nodes.

Case 3: $R_{s}<R\left(D_{0}\right)$ but not $R_{s} \ll R\left(D_{0}\right)$

The nearest node approximation in (17) works well in this case as well.

\section{CONCLUSION}

This paper analyzed the outage probability in cognitive radio networks, by classifying the typical outage events into three scenarios. When the average number of nodes in the forbidden region is much smaller than unity, the nearest node dominates the outage performance. When the average number of nodes in the forbidden region is greater than unity, the aggregate interference can be well approximated by a Gaussian random variable. When the average number of nodes in the forbidden region is slightly smaller than unity, neither the nearest node approximation nor the Gaussian one is accurate for the nonfading scenario, and the alternative approximation is accurate for the fading scenario.

\section{APPENDIX}

Sketch of the proof of (10) and (14): For (10), $\operatorname{Pr}\left\{d_{s}>D\right\}=\int_{0}^{\infty} f_{g s}(g) \bar{F}_{d}(D / g) d g$, where $\bar{F}_{d}(x)=$ $1-F_{d}(x)$ is the CCDF of $I_{a 1} / P_{0}$. Let $\bar{F}_{d}(D) \approx 1-$ $\exp \left(\bar{N}_{0} D_{\max }^{\frac{-2}{\nu}}\right)\left(1-\bar{N}_{0} D^{\frac{-2}{\nu}}\right)$. Taking the integral and splitting it into three parts: $\left[0, D / D_{\max }\right],\left[D / D_{\max }, D / D_{0}\right]$, and $\left[D / D_{0}, \infty\right]$, evaluating and approximating integrals for each interval, it can be shown that second integral is dominant and equals to (10). For (14), we use same approach except for $\bar{F}_{d}(D) \approx \bar{N}_{0} D^{\frac{-2}{\nu}}-\bar{N}_{0} D_{\max }^{\frac{-2}{\nu}}$.

\section{REFERENCES}

[1] Special Issue: "Cognitive Radio Part 2: Fundamental Issues", Proceedings of the IEEE, vol. 97, no.5, May 2009.

[2] E.S. Sousa and J.A. Silvester, "Optimum transmission ranges in a directsequence spread-spectrum multihop packet radio network," IEEE Journal on Selected Areas in Communications, vol.8, no.5, pp.762-771, Jun 1990.

[3] E.S. Sousa, "Performance of a spread spectrum packet radio network link in a Poisson field of interferers," IEEE Transactions on Information Theory, vol.38, no.6, pp.1743-1754, Nov 1992.

[4] J. Ilow and D. Hatzinakos, "Analytic alpha-stable noise modeling in a Poisson field of interferers or scatterers," IEEE Transactions on Signal Processing, vol.46, no.6, pp.1601-1611, Jun 1998.

[5] V. Mordachev and S. Loyka, "On node density - outage probability tradeoff in wireless networks," IEEE Journal on Selected Areas in Communications, vol.27, no.7, pp.1120-1131, September 2009.

[6] A. Ghasemi and E.S. Sousa, "Interference Aggregation in SpectrumSensing Cognitive Wireless Networks," IEEE Journal of Selected Topics in Signal Processing, vol.2, no.1, pp.41-56, Feb. 2008. 